\title{
The application of Expert System of Artificial Intelligence for advice in detection of disease in Health Care
}

\author{
*Mr. Rohit Kumar1 , *Dr. Amar Nath Singh² Member IEEE \\ * Master in Computer Application, Amity University, Jharkhand ${ }^{1}$ \\ * Dept. of Computer Science \& Engineering, Amity University, Jharkhand ${ }^{2}$
}

\begin{abstract}
:
In the present scenario the health is the prime concern for every of us. Due to the increase in pollution and the other contaminants in environmental atmosphere the chances of getting a disease is became a common factor. No, doubt we are very cautious to prevent ourselves but still due to because of busy schedule we can't able to take a proper precaution. Hence the medical science in association with modern technology and with the contribution of AI, has invented various gadgets using which we can take a precaution at earlier stages of disease. These devices are using the secure mode of data transmission for transferring the data in private mode. Here in this paper we are going to discuss the various aspect of medically analysis and detection of disease in Health care.
\end{abstract}

Keyword: Health care, precaution, Environments, Disease.

\section{Introduction:}

AI is getting increasingly sophisticated at doing what humans do, but more efficiently, more quickly and at a lower cost. The potential of Artificial Intelligence in healthcare is very vast. It has a great impact on our life schedule which includes our basic necessities goods may be a smart watch or as a computer. Just like in our every-day lives, AI in combination of robotics approach used as increasingly a part of our healthcare eco-system [3]. The schematic area of AI in addition with the robotic approach covers all most all area of science which is directly or indirectly helps us to improve the health sciences and detection of disease. In present scenario, the precaution factor for a human is very unpredictable, and hence, The AI with robotics application makes it simple as it is a small task. Now a day the medical science has improved a lot due to enhancement in technology, which is a blessing of AI.

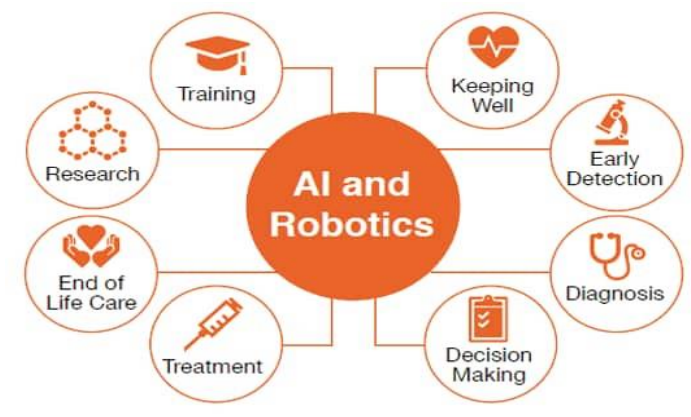

Fig-1 Schematic diagram for AI in Health Care
2. A precast diagnostic approach (Keeping Well): The AI technology has a great contribution to our life. One of AI's biggest potential benefits is to help people stay healthy so they don't need a doctor, or at least not as often [2]. The use of AI and the Internet of Medical Things (IoMT) in consumer health applications is already helping people up to great extent. AI increases the ability for healthcare professionals to better understand the day-to-day patterns and needs of the people they care for, and with that understanding they are able to provide better feedback, guidance and support for staying healthy.

Now days we have smart diagnostics machine which is capable enough to find the symptom and accordingly helps to suggest the precaution for the respective disease, which is refer as precast diagnostic approach or some time also called as Keeping Well technique [3].

The AI Technology applications and apps encourage healthier behaviour in individuals and help with the proactive management of a healthy lifestyle. It puts consumers in control of health and well-being.

\section{Early Detection:}

In lieu of detection of disease, The AI has a major role. It is smart and intelligent enough to take the decision and accordingly prescribe the remedy for the disease. If we look to the history of AI, then it has already being used to detect diseases, such as cancer, more accurately and in their early stages. According to the American Cancer Society, a high proportion of mammograms yield false results, leading to 1 in 2 
healthy women being told they have cancer. The use of $\mathrm{AI}$ is enabling review and translation of mammograms 30 times faster with $99 \%$ accuracy, reducing the need for unnecessary biopsies [1].

The proliferation of consumer wearable's and other medical devices combined with AI is also being applied to oversee early-stage heart disease, enabling doctors and other caregivers to better monitor and detect potentially life-threatening episodes at earlier, more treatable stages.

\section{Diagnosis:}

The IBM's Watson is a well known for healthcare organizations apply cognitive technology to unlock vast amounts of health data and power diagnosis. Watson can review and store far more medical information-every medical journal, symptom, and case study of treatment and response around the world - exponentially faster than any human [2].

Google's Deep Mind Health is working in partnership with clinicians, researchers and patients to solve realworld healthcare problems. The technology combines machine learning and systems neuroscience to build powerful general-purpose learning algorithms into neural networks that mimic the human brain.

\section{Decision Making:}

Decision Making is another important aspect of a machine which makes the machine more accurate and efficient. In the health care zone, the data are very vast and important because it dealt with real life. The Improving care requires the alignment of big health data with appropriate and timely decisions, and predictive analytics can support clinical decisionmaking and actions as well as prioritise administrative tasks. Due to the AI logic it makes the system more sophisticated and reliable in human prospect.

AI has a application area in pattern recognition which is used to identify patients at risk of developing a condition-or seeing it deteriorate due to lifestyle, environmental, genomic, or other factors - is another area where $\mathrm{AI}$ is beginning to take hold in healthcare.

\section{Treatment:}

Based on the pre casting and future analysis, the AI became more popular now a day. In the present scenario, it has influenced our medical science up to great extent. The scanning health records to help providers identify chronically ill individuals who may be at risk of an adverse episode, AI can help clinicians take a more comprehensive approach for disease management, better coordinate care plans and help patients to better manage and comply with their longterm treatment programmes [3].

Robots have been used in medicine for more than 30 years. They range from simple laboratory robots to highly complex surgical robots that can either aid a human surgeon or execute operations by themselves. In addition to surgery, they're used in hospitals and labs for repetitive tasks, in rehabilitation, physical therapy and in support of those with long-term conditions.

\section{Life care}

We are living much longer than previous generations, and as we approach the end of life, we are dying in a different and slower way, from conditions like dementia, heart failure and osteoporosis. It is also a phase of life that is often plagued by loneliness.

AI based Robots have the potential to revolutionise end of life care, helping people to remain independent for longer, reducing the need for hospitalisation and care homes. AI combined with the advancements in humanoid design are enabling robots to go even further and have 'conversations' and other social interactions with people to keep aging minds sharp [3].

\section{Research:}

The path from research lab to patient is a long and costly one. According to the California Biomedical Research Association, it takes an average of 12 years for a drug to travel from the research lab to the patient. Only five in 5,000 of the drugs that begin preclinical testing ever make it to human testing and just one of these five is ever approved for human usage. Furthermore, on average, it will cost a company US $\$ 359$ million to develop a new drug from the research lab to the patient [1].

Drug research and discovery is one of the more recent applications for AI in healthcare. By directing the latest advances in AI to streamline the drug discovery and drug repurposing processes there is the potential to significantly cut both the time to market for new drugs and their costs.

\section{Training:}

Training is another important factor, which is most of us should know so that we can use the device in a better sense. Mishandling of device may lead the erroneous result which interns may cause a death of the patients also. Hence, the knowledge is a must to handle the machine. Due to the advancement of AI, it became easier to handle such a vast device. The chances of error rate has also reduces up to great extent [3]. The AI allows those in training to go through naturalistic simulations in a way that simple computerdriven algorithms cannot. For example, The advent of natural speech and the ability of an AI computer to draw instantly on a large database of scenarios, means the response to questions, decisions or advice from a trainee can challenge in a way that a human cannot. And the training programme can learn from previous responses from the trainee, meaning that the challenges can be continually adjusted to meet their learning needs.

The training can be done anywhere; with the power of AI embedded on a smart phone, quick catch up 
sessions, after a tricky case in a clinic or while travelling, will be possible [1].

\section{Conclusion:}

Health is First. By looking to the above factors, In the present scenario the health is the prime concern for every of us. Due to the increase in pollution and the other contaminants in environmental atmosphere the chances of getting a disease is became a common factor. No, doubt we are very cautious to prevent ourselves but still due to because of busy schedule we can't able to take a proper precaution [2]. The AI is more robust and intelligent enough, but still in our country most of us don't know about the advance technology. Hence we need to educate the humans with proper sense of training. It makes the person aware about the pre-fore casting of symptoms about a disease and thus we can take proper precaution and treatment.

\section{About Author: \\ Er. Rohit Kumar is presently perusing the MCA at Amity University Jharkhand in the department of Information and Technology. \\ Dr. Amar Nath Singh presently the Prof. at Amity University Jharkhand in the department of Computer Science \& Engineering. His research areas are AI, WSN, Robotics and Data Science.}

\section{References:}

[1] California Biomedical Research Association. New Drug Development Process.

[2] The AI Industry Series: Top Healthcare AI Trends To Watch.

[3] AI And Healthcare: A Giant Opportunity. 